\title{
Generalizability of the Time-Lag Method for Mixed-Matrix Membranes: Does One Method Fit It All?
}

\author{
Haoyu Wu, Zheng Cao, Boguslaw Kruczek, Jules Thibault \\ Department of Chemical and Biological Engineering, University of Ottawa \\ 161 Louis Pasteur, Ottawa ON Canada, K1N 6N5, Ottawa, Canada \\ hwu055@uottawa.ca; zcao088@uottawa.ca; bkruczek@uottawa.ca; Jules.thibault@uottawa.ca
}

\section{Extended Abstract}

The time-lag method is a standard membrane characterization technique to determine the three transport coefficients diffusivity $(D)$, permeability $(P)$, solubility $(S)$ - from a single dynamic gas permeation test in a constant-volume system. A step-change in gas pressure on one side of the membrane initiates the dynamic experiment. Both sides of the membrane are initially exposed to a high vacuum. The experiment's progress is monitored by measuring the pressure of the permeating gas, which accumulates in a downstream chamber, while the pressure on the other side is maintained constant. The time lag $(\theta)$ is the intercept of the linear portion of the pressure vs time curve with the time-axis, and $D$ of the membrane is calculated from Eq. (1), which also requires the membrane thickness $(L)$.

$$
\boldsymbol{D}=\frac{\boldsymbol{L}^{2}}{6 \theta}
$$

The slope of the linear portion of the pressure response is directly proportional to $P$, and $S$ is the ratio of $P$ and $D$. Eq. (1) and the time-lag method are applicable for membranes made from rubbery polymers in which sorption is a linear function of the applied pressure. However, the time-lag method is extended to membranes made from glassy polymers, polymers with intrinsic microporosity, and mixed-matrix membranes (MMMs). It requires replacing $D$ in Eq. (1) with the effective diffusivity coefficient $\left(D_{\text {eff }}\right)$.

MMMs are the types of membranes comprised of inorganic fillers embedded in a continuous polymer matrix. Their properties are highly tunable by the properties and loading of inorganic particles. The improvement of MMMs fabrication protocols requires reliable expected permeation properties of the resulting membranes. The latter is obtained by numerical simulation of gas transport through ideal MMMs, assuming uniformly dispersed inorganic particles and no polymer-particle interface defects.

In our recent paper, we demonstrated that $D_{\text {eff }}$ of MMMs based on impermeable fillers determined using Eq. (1) might differ from $D_{\text {eff }}$ determined from $P$ and $S$ ratio [1]. The current work extends this investigation into MMMs with permeable inorganic fillers of different geometries, including cuboids, spheres, rods, and tubes. We allowed fillers' permeability coefficient $P_{p}$ to vary widely, from $10^{-3}$ to $10^{3}$ of the continuous polymer phase's permeability $(P)$. For shapes such as rods and tubes, we considered both horizontal and vertical orientations. Also, we considered the effect of the number of layers of which MMMs consist. In each layer, the permeating molecule encounters a single filler particle.

When the diffusivity coefficient in the filler $\left(D_{p}\right)$ is higher than in the polymer $(D)$, while the solubility coefficient in both phases is the same, $D_{\text {eff }}$ of the MMM is smaller than $D_{\text {eff }}$ determined from the $P$ and $S$ ratio. The opposite is true when the $D_{p}$ is lower than $D$. The more significant the difference between $D_{p}$ and $D$, the greater is the difference between the two $D_{\text {eff }}$ values. The size of the filler magnifies the difference between the two $D_{\text {eff }}$ values. The difference between $D_{\text {eff }}$ from the time lag and $P$ and $S$ ratio is most prominent in thin MMMs consisting of a single layer of particles. As the number of layers increases, the difference gradually decreases and practically disappears in MMMs consisting of five or more layers of particles, consistent with MMMs with impermeable membranes [1]. The main application of MMMs with impermeable particles is for barrier materials, for which thick multilayer MMMs are acceptable. However, when using highly permeable particles, the final MMMs should be as thin as possible, where the most significant discrepancy between the two $D_{\text {eff }}$ values exists.

\section{References}

[1] H. Wu, J. Thibault, B. Kruczek, "The validity of the time-lag method for the characterization of mixed-matrix membranes," J. Membr. Sci., vol. 618, 118715, 2021. https://doi.org/10.1016/j.memsci.2020.118715. 